\title{
When should we discontinue antiarrhythmic therapy for atrial fibrillation after coronary artery bypass grafting? A prospective randomized study
}

\author{
Uzi Izhar, MD \\ Niv Ad, MD \\ Ehud Rudis, MD \\ Eli Milgalter, MD \\ Amit Korach, MD \\ Nicola Viola, MD \\ Eli Levi, MD \\ Galit Asraff, RN, BSN \\ Gideon Merin, MD \\ Amir Elami, MD
}

From the Department of Cardiothoracic Surgery, Hadassah University Hospital, Jerusalem, Israel.

Received for publication March 17, 2004; revisions received June 10, 2004; accepted for publication June 28, 2004

Address for reprints: Uzi Izhar, MD, Cardiothoracic Surgery Department, Hadassah University Hospital, Jerusalem 91120, Israel (E-mail: izharu@bezeqint.net).

J Thorac Cardiovasc Surg 2005;129:401-6 $0022-5223 / \$ 30.00$

Copyright $\odot 2005$ by The American Association for Thoracic Surgery

doi:10.1016/j.jtcvs.2004.06.029
Background: New-onset atrial fibrillation after coronary artery bypass grafting is common. Medical therapy includes various antiarrhythmic drugs to control heart rate and restore sinus rhythm. The purpose of this study was to determine the duration of antiarrhythmic therapy after discharge from the hospital.

Methods: One hundred twenty-nine patients in whom new atrial fibrillation after coronary artery bypass grafting developed and successfully reverted to sinus rhythm were prospectively randomized at dismissal to receive antiarrhythmic therapy for 1 week (group $\mathrm{A} ; \mathrm{n}=44$ ), 3 weeks (group B; $\mathrm{n}=42$ ), or 6 weeks (group $\mathrm{C} ; \mathrm{n}=43$ ). Patients were followed up for an additional 4 weeks after discontinuation of antiarrhythmic therapy for detection of recurrent atrial fibrillation.

Results: The incidence of new atrial fibrillation during the study period was $21.2 \%$ (256/1206). Among the 129 patients who consented to the study, conversion to sinus rhythm was accomplished with the following medications: amiodarone (group A, $82 \%$; group $\mathrm{B}, 93 \%$; group $\mathrm{C}, 88 \% ; P=.29$ ), digoxin (group A, $16 \%$; group $\mathrm{B}, 7 \%$; group $\mathrm{C}, 7 \% ; P=.29$ ), $\beta$-blockers (group $\mathrm{A}, 27 \%$; group $\mathrm{B}, 19 \%$; group $\mathrm{C}, 14 \%$; $P=.30$ ), calcium channel blockers (group A, $2 \%$; group $\mathrm{B}, 2 \%$; group $\mathrm{C}, 0 \% ; P=$ .60 ), quinidine (group A, $2 \%$; group $\mathrm{B}, 2 \%$; group $\mathrm{C}, 7 \%$; $P=.44$ ), and procainamide (group $\mathrm{A}, 4.5 \%$; group $\mathrm{B}, 2 \%$; group $\mathrm{C}, 0 \% ; P=.37$ ). Follow-up was completed in 128 patients $(99.2 \%)$. There was no significant difference in the recurrence of atrial fibrillation among groups $(0 \%, 2 \%$, and $0 \%$ for groups $\mathrm{A}, \mathrm{B}$, and $\mathrm{C}$, respectively).

Conclusions: Patients with new atrial fibrillation after coronary artery bypass grafting, converted to normal sinus rhythm before hospital discharge, have a benign course. Antiarrhythmic therapy as short as 1 week may be appropriate in these patients.

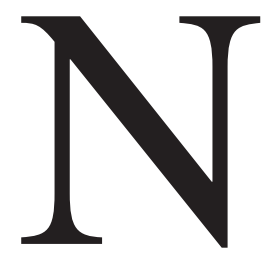
ew-onset atrial fibrillation (AF) after coronary artery bypass grafting (CABG) is common, with an incidence of up to one third of patients. ${ }^{1-3}$ Although postoperative atrial tachyarrhythmia is often regarded as a temporary problem related to the operation and therefore innocuous, this complication has clinically significant adverse effects on patient outcome. Post-CABG atrial arrhythmia is associated with a twofold increase in the duration of intensive care unit stay and 


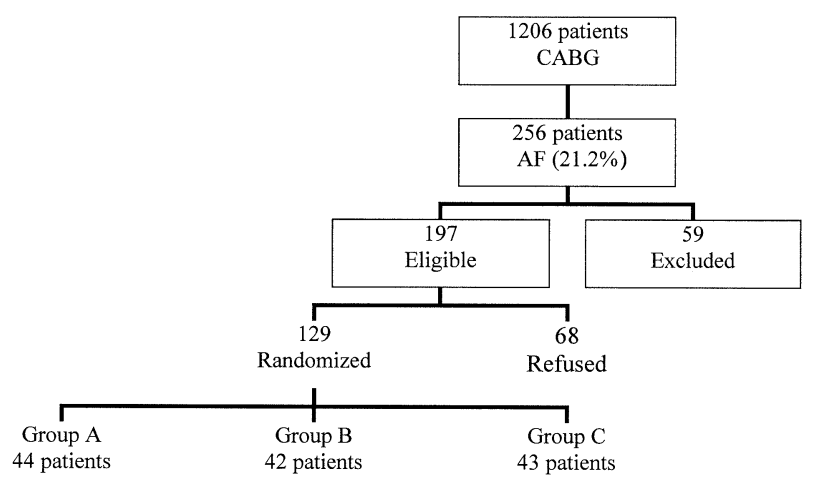

Figure 1. Study population. AF, Atrial fibrillation; $C A B G$, coronary artery bypass grafting.

a $50 \%$ to $70 \%$ prolongation of total hospitalization time with attendant increased hospitalization costs, and it is an important risk factor for perioperative cerebrovascular accidents. $^{4,5}$

Medical therapy includes various drugs, such as $\beta$-blockers, calcium channel blockers, digoxin, sotalol, amiodarone, quinidine, ibutilide, and procainamide, among others, to control heart rate and restore sinus rhythm. ${ }^{3}$ When postoperative AF is successfully converted to sinus rhythm, it is unlikely to recur, and nearly all of these patients are discharged from the hospital in sinus rhythm. ${ }^{1}$ Most of the antiarrhythmic agents have significant cardiac and noncardiac adverse effects. ${ }^{6}$ It is not clear how soon these patients may discontinue antiarrhythmic therapy to avoid these side effects without risking recurrence of AF. Current recommendations of the American Heart Association and the American College of Cardiology for the management of postoperative AF do not address the duration of antiarrhythmic therapy. ${ }^{7}$ We hypothesized that the course of antiarrhythmic therapy after CABG should be short and that there should be no difference in the recurrence of AF among groups of patients treated for different times. Therefore, we conducted this prospective randomized study among patients undergoing $\mathrm{CABG}$ in whom new-onset postoperative $\mathrm{AF}$ developed.

\section{Methods \\ Patient Selection}

From September 1, 1999, to May 31, 2003, 1206 patients underwent CABG at the Hadassah University Hospital, Jerusalem. We prospectively collected preoperative and postoperative clinical data on these patients. Inclusion criteria for participation in this study were (1) new-onset AF after CABG, (2) no history of atrial or ventricular arrhythmia, (3) no previous use of antiarrhythmic therapy, (4) normal sinus rhythm controlled with antiarrhythmic therapy by the time of hospital discharge, and (5) informed consent from each participant. Patients were excluded if they had a history of chronic or paroxysmal AF, if they took antiarrhythmic drugs before surgery, or if they were discharged with AF. Fifteen patients died after surgery and were excluded from the statistical analysis. AF developed in 256 patients (21.2\%) after surgery. In only 5 patients was sinus rhythm not restored, and they were discharged with AF. The success rate of drugs (247 patients) or electrical conversion (4 patients) was $98 \%$ (251/256). Fifty-nine patients (23\%) were excluded because they had a history of AF (37 patients), received antiarrhythmic therapy before surgery (18 patients), or were discharged with $\mathrm{AF}$ (5 patients). One hundred ninety-seven patients were eligible to participate in the study. Sixty-eight patients who fulfilled the inclusion criteria refused to participate in the study. The remaining 129 patients were randomized according to the study protocol (Figure 1), which was approved by the institutional review board.

\section{Study Protocol}

One hundred twenty-nine patients were included in the study. All patients were discharged with antiarrhythmic therapy to maintain sinus rhythm. On the day of discharge, patients were randomized into 1 of 3 therapy groups to receive antiarrhythmic therapy for 1 week (group A; 44 patients), 3 weeks (group B; 42 patients), or 6 weeks (group C; 43 patients). Patients who fulfilled the inclusion criteria but refused randomization to the study were discharged from the hospital with antiarrhythmic therapy for 6 weeks according to our nonstudy protocol. At the end of each study period (1,3, or 6 weeks), patients discontinued the antiarrhythmic therapy and underwent a 12-lead electrocardiogram (ECG) for rhythm documentation. All patients were followed up during their group time (1,3, or 6 weeks) and an additional 4 weeks for recurrence of significant arrhythmia or readmission for any reason. At the end of this 4 -week period, patients were requested to obtain a second 12-lead ECG. All ECGs and postoperative clinical data were prospectively collected. One (group C) patient was lost to follow-up and was excluded from the final analysis.

Patients who received preoperative $\beta$-blockers, providing that these drugs were not prescribed as an antiarrhythmic therapy, were included in the study, and there was no statistical difference in the number of these patients among the 3 groups (group A, 56.8\%; group B, 59.5\%; group C, 62.7\%). Patients continued to receive $\beta$-blockers after surgery as well. If postoperative AF occurred, they were treated with additional antiarrhythmic drugs. When these patients were included in the study, they were discharged with $\beta$-blockers plus the additional antiarrhythmic therapy. By the end of the randomized study duration $(1,3$, or 6 weeks), they discontinued only the additional antiarrhythmic drug.

\section{Operative Technique}

All operations were performed through a median sternotomy. A standard cardiopulmonary bypass technique was instituted in 106 (82\%) patients (35 in group A, 37 in group B, and 34 in group C; $P=.46$ ) by using aortoatrial cannulation with a roller pump at moderate hypothermia $\left(28^{\circ} \mathrm{C}-32^{\circ} \mathrm{C}\right)$. Myocardial protection was based on intermittent antegrade and retrograde tepid blood cardioplegia. Distal anastomoses were performed during a single period of aortic crossclamping, and proximal anastomoses were per- 
TABLE 1. Patient characteristics

\begin{tabular}{|c|c|c|c|c|}
\hline Variable & $\begin{array}{c}\text { Group A } \\
(1 \mathrm{wk} ; \mathrm{n}=44)\end{array}$ & $\begin{array}{c}\text { Group B } \\
\text { (3 wk; } n=42)\end{array}$ & $\begin{array}{c}\text { Group C } \\
(6 \mathrm{wk} ; n=43)\end{array}$ & $P$ value \\
\hline Age (y) & $66.80 \pm 8.73^{*}$ & $64.90 \pm 9.64$ & $67.56 \pm 8.38$ & .37 \\
\hline Male sex & $39(89)$ & $38(90.5)$ & $37(86)$ & .82 \\
\hline Smoking & $24(54.5)$ & $17(40.5)$ & $21(49)$ & .42 \\
\hline Diabetes mellitus & $16(36)$ & $11(26)$ & $16(37)$ & .49 \\
\hline Hypertension & $27(61)$ & $25(59.5)$ & $28(65)$ & .86 \\
\hline Hypercholesterolemia & $29(66)$ & $27(64)$ & $24(56)$ & .58 \\
\hline Renal failure & $1(2)$ & $1(2)$ & $4(9)$ & .21 \\
\hline History of CVA & $3(7)$ & $2(5)$ & $8(19)$ & .072 \\
\hline Previous MI & $19(43)$ & $18(43)$ & $21(49)$ & .82 \\
\hline Previous $\mathrm{PCl}$ & $13(29.5)$ & $8(19)$ & $6(14)$ & .19 \\
\hline Three-vessel disease & $34(77)$ & $37(88)$ & $31(72)$ & .45 \\
\hline $\mathrm{EF}<50 \%$ & $10(22.5)$ & $8(19)$ & $10(23)$ & .73 \\
\hline Urgent operation & $21(48)$ & $19(45)$ & $24(56)$ & .69 \\
\hline Use of CPB & 35 (79.5) & $37(88)$ & $34(79)$ & .46 \\
\hline No. distal anastomoses & $2.50 \pm 0.79$ & $2.88 \pm 0.97$ & $2.77 \pm 1.00$ & .15 \\
\hline Length of stay $(d)$ & $9.05 \pm 2.63$ & $8.98 \pm 2.46$ & $9.30 \pm 2.55$ & .85 \\
\hline
\end{tabular}

*Data are mean $\pm \mathrm{SD}$ or $\mathrm{n}(\%)$. CPB, Cardiopulmonary bypass; CVA, cerebrovascular accident; EF, ejection fraction; $M I$, myocardial infarction; $P C l$, percutaneous coronary intervention.

formed with partial aortic clamping during rewarming. Conduits for bypass included internal thoracic and radial arteries and saphenous veins. Twenty-three patients ( 9 in group A, 5 in group B, and 9 in group $\mathrm{C} ; P=.6$ ) underwent $\mathrm{CABG}$ with an off-pump technique (surgeon's preference).

\section{Management of Postoperative AF}

Patients did not receive prophylactic antiarrhythmic therapy during the preoperative or postoperative period. Management of postoperative AF consisted of (1) immediate heart rate control by intravenous calcium channel blockers; (2) conversion to sinus rhythm by using an intravenous loading dose of amiodarone $(5-7 \mathrm{mg} / \mathrm{kg}$ over 30-60 minutes, then $1 \mathrm{~g} / 24 \mathrm{~h}$ followed by oral maintenance [200 mg daily]); (3) using alternative antiarrhythmic therapy ( $\beta$ blockers, calcium channel blockers, digoxin, quinidine, and procainamide) for patients who were not responsive to amiodarone or had contraindications to its use (bradycardia or atrioventricular block); (4) direct current cardioversion for hemodynamically unstable patients or patients who were not responding to medical therapy; and (5) anticoagulation with intravenous heparin (monitored by partial thromboplastin time twice laboratory control values or higher) for patients who were not converted to sinus rhythm within 48 hours. Patients in whom sinus rhythm was restored were discharged without anticoagulation. All patients received daily aspirin $100 \mathrm{mg}$ for life.

\section{Follow-up}

A closed follow-up was designed for the patients included in this study. (1) They were telephoned during the time they were randomized $(1,3$, or 6 weeks) for confirmation that they were taking the antiarrhythmic therapy. (2) They received a second call by the day they discontinued therapy for confirmation that ECG recording would be obtained. (3) They received a third call after an additional 4 weeks for confirmation that a second ECG would be obtained. (4) They participated in an outpatient visit by the end of the follow-up period. (5) They were asked to inform us about any readmission or emergency department visits during the duration of the study. All medical records of readmission were collected. (6) A letter covering all the study details was sent to all family physicians caring for these patients.

\section{Statistical Analysis}

Data are expressed as absolute numbers or percentages and, where appropriate, as mean $\pm \mathrm{SD}$. Continuous variables were compared between groups by means of 1-way analysis of variance. Categorical variables were compared by means of $\chi^{2}$ or Fisher exact tests.

\section{Results}

\section{Demographics}

The mean age of the patients randomized to the study was $68.8 \pm 8.7$ years, $64.9 \pm 9.6$ years, and $67.5 \pm 8.3$ years in groups $\mathrm{A}, \mathrm{B}$, and $\mathrm{C}$, respectively $(P=.37)$. The patients' demographics and preoperative variables are presented in Table 1 . There were no statistically significant differences among groups.

\section{Postoperative AF}

The incidence of new-onset postoperative AF was 21.2\% (256/ 1206). The various antiarrhythmic drugs used among the 3 groups of patients are listed in Table 2. Most patients were converted to sinus rhythm with amiodarone (group A, 82\%; group $\mathrm{B}, 93 \%$; group $\mathrm{C}, 88 \% ; P=.29$ ). Other medications used to control AF included digoxin (group A, 16\%; group B, $7 \%$; group $\mathrm{C}, 7 \% ; P=.29$ ), $\beta$-blockers (group A, 27\%; group $\mathrm{B}, 19 \%$; group $\mathrm{C}, 14 \% ; P=.30$ ), calcium channel blockers 
TABLE 2. Antiarrhythmic therapy for postoperative atrial fibrillation

\begin{tabular}{|c|c|c|c|c|}
\hline Therapy & $\begin{array}{c}\text { Group A } \\
\text { (1 wk; } n=44)\end{array}$ & $\begin{array}{c}\text { Group B } \\
\text { (3 wk; } n=42)\end{array}$ & $\begin{array}{c}\text { Group C } \\
(6 \mathrm{wk} ; n=43)\end{array}$ & $P$ value \\
\hline Amiodarone & $36(82)$ & $39(93)$ & $38(88)$ & .29 \\
\hline$\beta$-Blockers & $12(27)$ & $8(19)$ & $6(14)$ & .30 \\
\hline Digoxin & $7(16)$ & $3(7)$ & $3(7)$ & .29 \\
\hline Calcium channel blockers & $1(2)$ & $1(2)$ & 0 & .60 \\
\hline Quinidine & $1(2)$ & $1(2)$ & $3(7)$ & .44 \\
\hline Procainamide & $2(4.5)$ & $1(2)$ & 0 & .37 \\
\hline
\end{tabular}

Data are $\mathrm{n}(\%)$.

(group A, 2\%; group B, 2\%; group C, $0 \% ; P=.60$ ), quinidine (group A, 2\%; group B, 2\%; group C, $7 \% ; P=.44$ ), and procainamide (group A, $4.5 \%$; group B, $2 \%$; group C, $0 \% ; P$ $=.37$ ). Direct current cardioversion was required in a small number of patients (group A, $2 \%$; group B, $2 \%$; group C, $5 \%$; $P=$.77).

AF-related complications included low cardiac output (group A, 0\%; group B, 4\%; group C, 0\%; $P=.10$ ) and requirement for anticoagulation therapy (group A, 21\%; group B, $17 \%$; group C, $12 \% ; P=.54)$. None of the patients had stroke.

\section{Follow-up and Recurrence of AF}

All patients were followed up for 4 weeks after discontinuation of medical therapy. Follow-up was completed in 128 patients (99.2\%): group A, 100\%; group B, 100\%; and group $\mathrm{C}, 98 \%(P=.82)$.

Recurrence of AF during the follow-up period was $0 \%$ in group A, $2 \%$ in group $\mathrm{B}$, and $0 \%$ in group $\mathrm{C}(P=.38)$. Only 1 patient in group B was readmitted for recurrent asymptomatic AF 1 week after he discontinued the treatment with amiodarone. He was successfully converted to sinus rhythm after resuming amiodarone therapy for another 4 weeks. None of the patients had stroke or episodes of transient ischemic attack during the follow-up period.

Eight patients were readmitted for various reasons: pneumonia, 1 patient; congestive heart failure, 2 patients; pleural effusion, 2 patients; and chest pain, arm wound infection, and AF, 1 patient each (group A, 4.8\%; group B, 9.5\%; group $\mathrm{C}, 5 \% ; P=.62)$.

\section{Discussion}

Atrial tachyarrhythmias may occur in $25 \%$ to $40 \%$ of patients undergoing $\mathrm{CABG}$, with a peak incidence between the second and fourth days after surgery. Among the patients who have no history of atrial tachyarrhythmias, spontaneous conversion of AF has been reported in $15 \%$ to $30 \%$ within 2 hours. 8,9

The precise cause and mechanism of the development of postoperative AF has yet to be established. It involves reentry, resulting from dissimilar refractoriness between adjacent atrial areas, without a known reason. ${ }^{10}$ Risk factors associated with the development of postoperative AF include advanced age, prolonged preoperative atrial conduction, atrial myolysis, chronic obstructive pulmonary disease, excess circulating catecholamines, electrolyte imbalance, atrial myocardial ischemia, $\beta$-blocker withdrawal, prolonged aortic crossclamping time, and right atrial manipulations. ${ }^{1-3}$

The principles of treatment for new-onset postoperative AF are similar to those for atrial tachyarrhythmias in other clinical circumstances. ${ }^{11}$ Mainly, 2 management strategies are available to treat patients with persistent AF: rate control and rhythm control. ${ }^{12}$ Although the rate-control strategy is preferred for patients in whom restoration of sinus rhythm is less important, most patients with new-onset postoperative AF are treated with antiarrhythmic therapy. ${ }^{13}$ A recent prospective randomized study comparing primary rate control with conversion strategy in postoperative AF showed little difference in time to conversion between the 2 groups of patients. ${ }^{9}$ This study also did not show a statistically significant reduction in the total length of stay through the conversion strategy, although the time from operation to discharge was significantly decreased. ${ }^{9}$ The control of ventricular rate, conversion to sinus rhythm, maintenance of regular rhythm, and anticoagulation to protect against thromboembolic complications are the goals of therapy. ${ }^{11}$

When should we discontinue antiarrhythmic therapy in patients undergoing $\mathrm{CABG}$ in whom new-onset postoperative AF develops? The treatment of patients with postoperative AF has not been standardized despite large clinical experience and research. ${ }^{14}$ There are few data regarding the post-hospital discharge course of patients with this complication. ${ }^{15,16}$ It is generally believed that there is no need for long-term suppressive therapy because in patients without a history of atrial tachyarrhythmia, once AF is converted to sinus rhythm, it is unlikely to recur. ${ }^{6}$ However, one of the fundamental questions regarding antiarrhythmic therapy for postoperative AF is whether any therapy should be given at all, once patients are discharged home already in sinus 
rhythm. We searched the medical literature for guidelines or studies addressing no additional antiarrhythmic therapy at discharge, but we could not find this practice as evidence based. We have demonstrated in this study that the duration of antiarrhythmic therapy in these patients may be very short, from 1 to 3 weeks, no matter what antiarrhythmic drugs have been used. There was no significant difference in the recurrence of AF during the follow-up period among the different therapy groups.

Our results are consistent with the very few studies reporting the frequency of recurrent $\mathrm{AF}$ after reversion or conversion to sinus rhythm after surgery. Kowey and associates $^{15}$ recently reported a retrospective analysis of the intermediate prognosis of patients undergoing $\mathrm{CABG}$ who had been treated with different strategies for postoperative AF. They concluded that the rate of recurrence of AF after discharge was similar in patients receiving class I or class III antiarrhythmic drugs together with rate-control agents and in those receiving rate-control drugs alone. Landymore and Howell ${ }^{16}$ reported on the recurrence of postoperative atrial arrhythmia in patients who had been treated with digoxin for 3 to 8 weeks after CABG. Their data indicated that AF rarely recurred after hospital discharge and was never symptomatic. They concluded that patients in whom new-onset postoperative AF developed should be treated with digoxin for 3 weeks after operation and that drug therapy should then be discontinued indefinitely. Yilmaz and associates ${ }^{17}$ have found that placebo was comparable to any medication in preventing the recurrence of $\mathrm{AF}$ after CABG: the relapse rate within 90 days of operation was 1 of 30 taking placebo, 2 of 30 taking quinidine, 2 of 30 taking verapamil, and 2 of 30 taking amiodarone.

Most of the patients in our study were successfully treated with amiodarone to control $\mathrm{AF}$ and maintain sinus rhythm in the postoperative period. Amiodarone, a class III antiarrhythmic agent, increases the refractory period of atrial and ventricular muscle as well as the atrioventricular node. In addition, it has mild $\beta$-blocker and calcium channel blocker activity. It has been reported that the drug is moderately effective for pharmacologic cardioversion of recentonset $\mathrm{AF} .{ }^{18}$ The conversion rate in patients with $\mathrm{AF}$ longer than 7 days is limited. Amiodarone also has been proven effective for conversion of persistent AF. ${ }^{19}$ A meta-analysis of 91 trials not specifically related to surgery concluded that the relative efficacy of the most commonly used antiarrhythmic drugs seems similar. ${ }^{20}$ Class IA (quinidine, procainamide, and disopyramide), class IC (flecainide and propafenone), and class III (amiodarone, sotalol, ibutilide, and dofetilide) drugs were all more effective than placebo, converting $40 \%$ to $60 \%$ of the patients compared with $30 \%$ with placebo. Comparisons of class IA or class IC with class III drugs revealed no significant differences. In deciding the nature and duration of drug therapy for postoperative $\mathrm{AF}$, we should consider not only the efficacy of the various antiarrhythmic drugs, but also their undesirable effects. Many previous studies have addressed the increased risk for proarrhythmias-specifically ventricular tachycardia and ventricular fibrillation - when using class I drugs to prevent recurrence of AF. ${ }^{6}$ Among the other serious side effects of this class of drugs are allergic/immune reactions (lupus-like syndrome), thrombocytopenia, myocardial depression, and gastrointestinal disturbance. Amiodarone, a class III antiarrhythmic drug, has been reported to be effective in preventing and treating postoperative $\mathrm{AF}^{21,22}$ The dose regimen used in our patients did not result in important side effects, and no-drug related complications were reported during the follow-up period. The half-life of amiodarone is prolonged, ranging from 26 to 107 days. ${ }^{23}$ Therefore, it is likely that once the patient with postoperative AF is loaded with amiodarone and converted to sinus rhythm, he or she may gain its persistent antiarrhythmic effect for several weeks. ${ }^{22,23}$ This strengthens our conclusion that the antiarrhythmic therapy after postoperative AF should be brief.

Twenty-four patients from the study population were operated on with the off-pump technique. Recent data indicate that these patients have a similar or lower incidence of postoperative AF than patients with the on-pump technique. ${ }^{24-26}$ Our incidence of postoperative $\mathrm{AF}$ for both groups was not statistically different. Therefore, the offpump patients who met the criteria of this study were eligible to be included in this trial, and both groups were analyzed together.

\section{Limitations of the Study}

Holter monitoring was not performed to screen for postoperative $\mathrm{AF}$ or other arrhythmia during the postoperative follow-up period. Instead, patients were asked to obtain a 12-lead ECG at the day therapy was discontinued and 4 weeks later. All readmission records were also evaluated for recurrent arrhythmia. The primary goal of the study was to determine when to discontinue the antiarrhythmic therapy for postoperative AF. We believe that failure to detect brief asymptomatic episodes of AF is unlikely to affect patient care or affect it adversely and that this does not detract from the clinical implication of this study.

\section{Conclusions}

Postoperative atrial arrhythmia remains a vexing clinical problem, the therapy of which has not yet been standardized. We conclude from our prospective randomized study that antiarrhythmic therapy for postoperative $\mathrm{AF}$ in patients undergoing CABG should be brief. A 1- to 3-week period is safe and sufficient in most patients.

\section{References}

1. Ommen SR, Odell JA, Stanton MS. Atrial arrhythmia after cardiothoracic surgery. $N$ Engl J Med. 1997;336:1429-34. 
2. Hogue CW, Hyder ML. Atrial fibrillation after cardiac operation: risks, mechanisms, and treatment. Ann Thorac Surg. 2000;69:300-6.

3. Maisel WH, Rawn JD, Stevenson WG. Atrial fibrillation after cardiac surgery. Ann Intern Med. 2001;135:1061-73.

4. Creswell LL, Schussler RB, Rozenbloom M, Cox JL. Hazards of postoperative atrial arrhythmia. Ann Thorac Surg. 1993;56:539-49.

5. Almassi GH, Schwalter T, Nicolasi AC, Aggarwal A, Moritz TE, Henderson WG, et al. Atrial fibrillation after cardiac surgery: a major morbid event? Ann Surg. 1997;226:501-13.

6. Humphries JO. Unexpected instant death following successful coronary artery bypass graft surgery (and other clinical settings): atrial fibrillation, quinidine, procainamide, et cetera, and instant death. Clin Cardiol. 1998;21:711-8.

7. ACC/AHA/ESC guidelines for the managements of patients with atrial fibrillation. J Am Coll Cardiol. 2001;38:1266li-lii.

8. Soucier RJ, Mizra S, Abordo MG, Berns E, Dalamagas HC, Hanna A, et al. Predictors of conversion of atrial fibrillation after cardiac operation in the absence of class I or III antiarrhythmic medications. Ann Thorac Surg. 2001;72:694-8.

9. Lee JK, Klein GJ, Krahn AD, Yee R, Zarnke K, Simpson C, et al. Rate-control versus conversion strategy in postoperative atrial fibrillation: a prospective randomized pilot study. Am Heart J. 2000;140: 871-7.

10. Cox JL. A perspective of postoperative atrial fibrillation in cardiac operation. Ann Thorac Surg. 1993;54:405-9.

11. Falk RH. Atrial fibrillation. N Engl J Med. 2001;344:1067-78.

12. Van Gelder IC, Hagens VE, Bosker HA, Kingma JH, Kamp O, Kingma T, et al. A comparison of rate control and rhythm control in patients with recurrent persistent atrial fibrillation. $N$ Engl J Med. 2002;347:1834-40.

13. Jayam VKS, Flaker GC, Jones JW. Atrial fibrillation after coronary bypass: etiology and pharmacological prevention. Cardiovasc Surg. 2002;10:351-8.

14. Crystal E, Connolly SJ, Sleik K, Ginger TJ, Yusuf S. Intervention on prevention of postoperative atrial fibrillation in patients undergoing heart surgery. A meta-analysis. Circulation. 2002;106:75-80.

15. Kowey PR, Stebbins D, Igidbashian L. Clinical outcome of patients who developed paroxysmal atrial fibrillation after CABG surgery. Pacing Clin Electrophysiol. 2001;24:191-3.
16. Landymore RW, Howell F. Recurrent atrial arrhythmias following treatment for postoperative atrial fibrillation after coronary bypass operations. Eur J Cardiothorac Surg. 1991;5:436-9.

17. Yilmaz AT, Demirkilic U, Arslan M, Kurulay E, Ozal E, Tatar H, et al. Long-term prevention of atrial fibrillation after coronary bypass surgery: comparison of quinidine, verapamil, and amiodarone in maintaining sinus rhythm. J Card Surg. 1996;11:61-4.

18. Galve E, Rius T, Ballester R, Artaza MA, Arnau JM, Garcia-Dorado $\mathrm{D}$, et al. Intravenous amiodarone in treatment of recent-onset atrial fibrillation: results of a randomized, controlled study. J Am Coll Cardiol. 1996;27:1079-82.

19. Kochiadakis GE, Igoumenidis NE, Solomou MC, Kaleboubas MD, Chlouverakis GI, Vardas PE. Efficacy of amiodarone for the termination of persistent atrial fibrillation. Am J Cardiol. 1999;83:58-61.

20. Nichol G, McAlister F, Pham B, Laupacis A, Shea B, Green M, et al. Meta-analysis of randomized controlled trails of the effectiveness of antiarrhythmic agents at promoting sinus rhythm in patients with atrial fibrillation. Heart. 2002:87:535-43.

21. Yagdi T, Nalbantgil S, Ayik F, Apaydin A, Islamoglu F, Posacioglu H, et al. Amiodarone reduces the incidence of atrial fibrillation after coronary artery bypass grafting. J Thorac Cardiovasc Surg. 2003;125: 1420-5.

22. Daoud EG, Strickberger A, Ching Man K, Goyal R, Deeb GM, Bolling $\mathrm{SF}$, et al. Preoperative amiodarone as prophylaxis against atrial fibrillation after heart surgery. $N$ Engl J Med. 1997;337:1785-90.

23. Hondeghem LM. Class III agents: amiodarone, bretylium, and sotalol. In: Zipes DP, Jalife J, editors. Cardiac electrophysiology: from cell to bedside. 2nd ed. Philadelphia: WB Saunders; 1995. p. 1330-6.

24. Hakala T, Pitkanen O, Hartikainen J. Cardioplegic arrest does not increase the risk of atrial fibrillation after coronary artery bypass surgery. Eur J Cardiothorac Surg. 2004;25:415-8.

25. Legare JF, Buth KJ, King S, Wood J, Sullivan JA, Friesen CH, et al. Coronary bypass surgery performed off pump does not result in lower in-hospital morbidity than coronary artery bypass grafting performed on pump. Circulation. 2004;109:887-92.

26. Mack MJ, Pfister A, Bachand D, Emery R, Magee MJ, Connolly M, et al. Comparison of coronary bypass surgery with and without cardiopulmonary bypass in patients with multivessel disease. $J$ Thorac Cardiovasc Surg. 2004;127:167-73. 